\title{
Accurate Detection and Characterization of Corner Points Using Circular Statistics and Fuzzy Clustering
}

\author{
M. E. Díaz, G. Ayala, J. Albert, F. J. Ferri and J. Domingo \\ Universitat de València, Institut de Robòtica, Dr. Moliner 50, \\ E-46100 Burjassot, Spain \\ elena.diaz@uv.es
}

\begin{abstract}
Accurate detection and characterization of corner points in grey level images is considered as a pattern recognition problem. The method considers circular statistic tests to detect $2 \mathrm{D}$ features. A fuzzy clustering algorithm is applied to the edge orientations near the prospective corners to detect and classify them. The method is based on formulating hypotheses about the distribution of these orientations around an edge, corner or other 2-D feature. The method may provide accurate estimates of the direction of the edges that converge in a corner, along with their confidence intervals. Experimental results show the method to be robust enough against noise and contrast changes. Fuzzy membership improves the results of the algorithm and both versions (crisp and fuzzy) give better results than other previously proposed corner detectors.
\end{abstract}

\section{Introduction}

Feature detection and, in particular, relevant point detection in images plays an important role in a number of tasks in computer vision. Most local features used in object recognition are related to the curvature of edges or surfaces. Examples of these local features are edges, corners, segments, and centers of circular symmetries. To name just a few examples, such points can be used to determine the position and orientation of objects for 3D reconstruction, or to select important parts of objects for tracking applications, or even to appropriately perform feature-based image matching in motion or stereo applications [1].

Corner or junction points are important local 2D features from this family. Conventionally, a corner point is defined as the intersection between two or more straight line edges with different orientations. From a more general point of view they can also be defined as high curvature points. An obvious method to detect corners follows then from curvature estimation techniques applied after obtaining an edge image [2-5]. The main drawback of this kind of methods comes from the fact that the final result strongly depends on the accuracy of the edge detector and the poor support to calculate the edge orientations specifically at corner

This work was partially supported by the Spanish CICYT project TIC95-676-C0201 . 
positions. If only straight lines or segments are expected in the image, corners can be obtained through line fitting in a robust way [1] but, unfortunately, this is seldom the case in most real world applications. Nevertheless, some approaches are related to this idea but applied in a local way [6].

Most of the approaches above mentioned are referred to as grey level corner detectors because they are applied directly to the grey level image without any previous application of other low-level processing or segmentation techniques in which arcs or curves have to be explicitly obtained. The main disadvantages of the techniques, that need a prior segmentation step, are that their performance depends on the success of this step and also that, as the approach is inherently sequential, the computational cost is usually larger.

In this paper, a recently introduced gray-level 2-D feature detector, based on a statistical analysis of local gradient orientations [7] is improved to accurately detect corner points in a variety of situations. The main contribution of this work consists of using a fuzzy clustering algorithm to appropriately obtain the parameters (orientations and confidence intervals) of the different edges at a corner point. As the corresponding crisp version, the method can be implemented in a computationally inexpensive way and is more robust to noise than other corner detectors previously proposed. By its nature, the method considered in this paper can discriminate among different types of corners ( $n$-junctions) and also it can accurately compute the orientations (and associated errors) of each edge in a junction.

The paper is organized as follows. In the next section, the foundation of the method for corner detection based on circular statistics is outlined. In Section 3, the particular method to segment different orientations by means of fuzzy clustering is described. The complete algorithm proposed is explained in Section 4 and different sets of comparative experiments considering other comer detectors $[2-4,6]$ apart from both crisp and fuzzy versions of the algorithm presented are included in Section 5. Finally, the main conclusions about the work presente are briefly explained in Section 6 .

\section{Modelling a Corner by Using Gradient Orientations}

Let $f(p)$ be the gray level observed at pixel $p$ in a given image. The smoothed gradient image, $\nabla_{G} f(p)$, is computed in the usual way by using the gradient and Gaussian smoothing operators. The thresholded gradient image, $g$, is then defined at the pixel $p$ as follows:

$$
g(p)= \begin{cases}\nabla_{G} f(p) & \text { if }\left|\nabla_{G} f(p)\right| \geq E, \\ 0 & \text { otherwise }\end{cases}
$$

where $E$ is a given threshold. Let $S_{g}$ be the support set of $g$, i.e., the set of pixels $p$ where $g$ is nonnull. The orientation of the gradient, $\theta_{p}$, is computed at each pixel $p$ in $S_{g}$. Finally, for each pixel $p$ in $S_{g}$, let $N(p)$ be a neighbourhood of $p$ defined in such a way that $N(p) \subset S_{g}$. 
An edge point can be defined as a pixel $p$ of the image whose gradient, $g(p)$, is nonnull and whose observed orientations $\theta_{q}$ for all $q$ in $N(p)$ are similar. These orientations should be compacted around a unique direction, $\mu$. Noise from the original image and the estimation procedure produce stochastic variation around the unknown parameter $\mu$. To account for this natural variability of the local orientations of the gradient image, $g(p)$, a reasonable assumption is to assume that $\theta$ follows a von Mises distribution [8] with probability density function:

$$
h(\theta)=\frac{1}{2 \pi I_{0}(\kappa)} \exp (\kappa \cos (\theta-\mu))
$$

where $\mu$ is the mean orientation and $\kappa$ is a parameter of concentration around $\mu . I_{0}(\kappa)$ denotes the Bessel function of first type of imaginary argument and is a normalisation factor.

A corner point can be defined as a pixel $p$ in the image whose gradient is nonnull and whose orientations $\theta_{q}$ for all $q$ in $N(p)$ are grouped around two or more different modes. These modes correspond to directions orthogonal to the orientation of the different edges that meet at the corner. In this case the variability of the local orientations can be modelled as a mixture of $n$ von Mises distributions as follows:

$$
h(\theta)=\sum_{i=1}^{n} \frac{p_{i}}{2 \pi I_{0}\left(\kappa_{i}\right)} \exp \left(\kappa_{i} \cos \left(\theta-\mu_{i}\right)\right) \quad \text { with } n \geq 2,
$$

where $\mu_{i}$ and $\kappa_{i}$ are defined for each component of the mixture, $n$ is the number of edges meeting at the corner and $p_{i}$ is the mixing proportion of the $i$-th population.

If the properties of each meeting edge (basically its orientation) have to be obtained from the gradient orientation, the above formulation may give problems in some cases because edge and gradient orientations differ in $\pm \frac{\pi}{2}$ depending on the kind of edge (dark-to-bright or bright-to-dark). Type $T$ junctions may also give problems because only two different gradient orientations will be obtained in these cases.

To overcome these problems, instead of considering gradient orientations, it is better to consider edge orientations in the neighbourhood $N(p)$ with respect to the central point, $p$. In this way, the orientations considered are independent of the kind of edge and also, the directions of the three edges converging in a $T$ junction will be correctly detected.

For specific applications in which edge orientations at a corner are not needed, the gradient orientations may be used. Also, orientations in the range 0 to $\pi$ (or even in any other range from 0 to $\pi / \ell$ ) may be considered in special cases by using a von Mises type angular distribution [8, page 68,69$]$. In the current application, the range $[0,2 \pi)$ is used to accurately detect the orientation of the different edges meeting at a corner point. 


\section{Parameter Estimation and Corner Classification}

The problem of corner detection and classification can be transformed into a pattern recognition problem under the assumptions made in the previous section. Once the hypothesis of having mixtures of von Mises distributions to model edge orientations has been assumed, it can be easily tested the goodness-of-fit of the corresponding distribution.

\subsection{Detecting Edge Points}

The parameters $\mu$ and $\kappa$ of a von Mises distribution can be estimated using a maximum likelihood approach [9]. In particular, the average direction can be estimated as

$$
\hat{\mu}=\arctan \frac{\sum_{j=1}^{m} \sin \theta_{j}}{\sum_{j=1}^{m} \cos \theta_{j}} .
$$

To obtain an estimate of $\kappa$, the peakness factor, $R$, is computed first as

$$
R=\left[\left(\sum_{j=1}^{m} \sin \theta_{j}\right)^{2}+\left(\sum_{j=1}^{m} \cos \theta_{j}\right)^{2}\right] / m
$$

where $m$ is the number of points in the neighbourhood $N(p)$. The estimate $\hat{\kappa}$ is then obtained in terms of $R$ [10]. The computation of $\hat{\kappa}$ is especially straightforward in the (interesting) case in which $R>0.9$ which corresponds to a sufficiently peaked distribution as usually occurs at corners.

Once the parameters $\mu$ and $\kappa$ have been estimated, the Watson-Stephens test [8] is applied to test the hypothesis that the data fits the von Mises density of Equation 2. This test is easy to implement, powerful enough and can be applied to any kind of density function.

In this way, edge points can be robustly estimated by considering edge orientations in a convenient neighbourhood. The parameter $\kappa$ is needed to perform the test but also, its value may give us an indication of the dispersion of the local orientations in the edge because it provides in fact a confidence interval for the estimated orientations in $N(p)$ [10].

\subsection{Detecting Corner Points}

In the case of a mixture of $n \geq 2$ components there are no analogous expressions to estimate the corresponding parameters. In principle, an iterative method which maximizes the likelihood could be proposed for parameter estimation as in the case of normal mixtures [11]. Nevertheless, a more straightforward approach has been adopted to separately estimate the parameters of each component [7]. Since in the particular case of edge orientations for corner detection the components of a mixture are usually sufficiently separated and peaked, the corresponding orientations in $N(p)$ can be conveniently assigned to different edges by 
means of a clustering process. More concretely, to test if a particular sample follows a von Mises distribution consisting of $n$ components, a $n$-means clustering algorithm is applied and then a separate maximum likelihood estimation of $\mu_{i}$ and $\kappa_{i}$ for each component $i$ is performed as in the case of a unimodal von Mises distribution [7].

As each cluster is taken to be an individual von Mises distribution whose parameters have to be estimated, any misclassification at the overlapping regions between components will give rise to an increment of the error of the estimator of $\mu_{i}$. To partially overcome this effect, a fuzzy clustering approach is proposed in this work in such a way that samples in the overlapping regions partially belong to several components. In this way, the (fuzzy) splitting of components represent in a more accurate way the true density function in Equation 3 because the tails of each component are partially taken into account.

In this paper, the fuzzy $n$-means algorithm [12] has been used in order to obtain the corresponding fuzzy partition of the orientations in $N(p)$. In this case, to obtain the average direction of each component, the membership values are used to weight the contribution of each sample in Equation 4 which gives rise to a straightforward fuzzy version of estimator as:

$$
\hat{\mu_{i}}=\arctan \frac{\sum_{j=1}^{m} u_{i j} \cdot \sin \theta_{j}}{\sum_{j=1}^{m} u_{i j} \cdot \cos \theta_{j}},
$$

being $u_{i j}$ the fuzzy membership value of the $j$-th element to the $i$-th component.

In the same way, the corresponding expression for $R$ follows from Equation 5 .

$$
R_{i}=\left[\left(\sum_{j=1}^{m} u_{i j} \cdot \sin \theta_{j}\right)^{2}+\left(\sum_{j=1}^{m} u_{i j} \cdot \cos \theta_{j}\right)^{2}\right] /\left(\sum_{j=1}^{m} u_{i j}\right) .
$$

In this equation, the number of samples in a component is substituted by the fuzzy cardinality of the corresponding component.

The Watson-Stephens test can be used again to test if the orientations in $N(p)$ follow the density in Equation 3 with the parameters $\mu_{i}$ and $\kappa_{i}$ obtained from the clustering process. The parameter $p_{i}$ could be obtained from the previous clustering. Also, in the particular case of using circular neighbourhoods, it can be assumed that at any $n$-junction, $p_{i}$ equals $1 / n$.

\section{Algorithm for 2D Feature Detection}

As currently implemented, the algorithm works by sequentially testing different null hypotheses. The first one corresponds to testing if the gradient orientations follow a von Mises distribution, with parameters $\mu$ and $\kappa$ estimated from the data, using the Watson-Stephens test [13]. That means detecting edge points with direction perpendicular to $\hat{\mu}$ when the hypothesis cannot be rejected. The algorithm goes to the next prospective point if this occurs.

If the first test fails, the algorithm then starts by considering mixtures of von Mises distributions from $n=2$ to a maximum value ( 4 in the experiments 
presented here). For each value of $n$ the hypothesis "the orientations follow the density in Equation 3" is tested. As soon as a hypothesis cannot be rejected, the algorithm goes to the next pixel. Otherwise, the algorithm will proceed to the next value of $n$. As in this case, a number of successive different tests are applied using the same data, a Bonferroni's correction to the significance level used in the test is applied.

The hypotheses have to be applied for pixels in $S_{g}$ only. In the worst case, $n$ different tests are applied at the same location. Note that this is the most uncommon case, since the biggest part of points are edge-points and they pass the first test.

Apart from applying all this to prospective points to detect corners, it is convenient to perform a final non-maxima suppression to keep only those points which have the highest significance level of the accepted hypothesis in a certain neighbourhood. This comes from the fact that there cannot be two or more corner points in a sufficiently small neighbourhood. The whole algorithm is presented in Figure 1.

Step 0 (Initialisation) Fix a threshold level $E$, a maximum value $n_{\text {max }}$, a significance level for the tests $\alpha_{0}$, a size and shape for the neighbourhood $N(p)$ and a minimal distance between corner points for non-maxima suppression. Compute the image $g$ (Equation 1) and estimate the orientation of the gradient at every point $p \in S_{g}$.

Apply steps 1 to 7 to each $p \in S_{g}$ in the image.

Step 1. estimate $\mu$ and $\kappa$ using Equations 4 and 5 .

Step 2. test the null hypothesis "the distribution of gradient orientations in $N(p)$ follow a von Mises distribution with parameters $(\mu, \kappa)$ ".

Step 3. if the hypothesis cannot be rejected at significance level $\alpha_{0}$ then $p$ is an edge point with orientation orthogonal to $\mu$. Go to the next pixel, else, go to step 4 .

Step 4. $n \leftarrow 2, \alpha \leftarrow \alpha_{0} / 2$

Step 5. estimate $\left(\mu_{i}, \kappa_{i}\right)$ for $i=1 \ldots n$ assuming that the edge orientations in $N(p)$ follow a mixture of $n$ components by using the fuzzy $n$-means algorithm and Equations 6 and 7 .

Step 6. test the null hypothesis "the distribution of the edge orientations in $N(p)$ is a mixture of von Mises distributions with parameters $\left(\mu_{i}, \kappa_{i}\right)$ $i=1 \ldots n "$.

Step 7. if the hypothesis cannot be rejected at significance level $\alpha$, then $p$ is a corner point where $n$ edges converge. Go to the next pixel.

else $n \leftarrow n+1$ and $\alpha \leftarrow \alpha_{0} / n$. Go to step 5 if $n \leq n_{\max }$.

Step 8. (End) Do a final non-maxima suppression to eliminate too close detected corners.

Fig. 1. Algorithm for corner detection and classification. 
The neighbourhood has been selected to be circular in such a way that $N_{M}(p)=\left\{q \in S_{g}: d(p, q) \leq M\right\}$ where $d(\cdot, \cdot)$ stands for the Euclidean distance. As $N_{M}(p)$ has to be large enough so as to contain a sufficient number of points in $S_{g}$ for the tests to be applied but also sufficiently small to keep the main assumptions about corner modelling correct, a value $M=12$ has been selected. The minimal distance between corner points for non-maxima suppression depends on the application, a value of 15 pixels has been taken in these cases. Initial value for the significance level $\alpha_{0}$ was 0.05 and for the threshold level $E$ was 100 . The gradient image was normalized in the $[0, \ldots, 255]$ range.

\section{Experimental Results}

A number of experiments using crisp and fuzzy versions of the clustering algorithm of the proposed method have been carried out considering both synthetic and real images.

First, to test the effectiveness of the proposed algorithm from a numerical point of view, a simulation has been conducted using a synthetic image containing $L, X, Y$ and + junctions (see Figure 2) introduced by Cooper et al.[4]. The contrast of the synthetic image is reduced progressively from top to bottom. The image is then smoothed by convolution with a Gaussian kernel to simulate the blurring introduced by the acquisition system, and then Gaussian noise is added to test the robustness. To compare the performance of the corner detector, the criterion of Cooper et al. is used: a $5 \times 5$ detection area centered at a true corner is examined. Any corner detected outside this region is termed as false positive (FP), and failure to detect an existing corner in the detection area is termed as false negative (FN).

The results obtained by the proposed methods with both crisp and fuzzy clustering versions lead to the same detection results. Table 1 shows FN's and FP's obtained for the synthetic image. This table shows that the proposed method gives better results than any other analyzed and is more robust to Gaussian noise; dashes represent data not given in the bibliography.

Table 1. Error performance of operators in terms of False Positives (FP) and False Negatives (FN)

\begin{tabular}{|c|c|c|c|c|c|c|c|c|}
\hline $\begin{array}{l}\text { Image } \\
\text { noise }\end{array}$ & $\begin{array}{l}\text { Cooper } \\
\text { FP FN }\end{array}$ & & $\begin{array}{l}\text { Kit } \\
\text { FP }\end{array}$ & $\begin{array}{l}\text { Ros } \\
\text { FN }\end{array}$ & $\begin{array}{l}\text { Bea } \\
\text { FP }\end{array}$ & FN & FP & $\begin{array}{l}\text { Det. } \\
\text { FN }\end{array}$ \\
\hline$\sigma=6$ & 1718 & $\begin{array}{ll}18 & 44\end{array}$ & 17 & 33 & 18 & 14 & 0 & 0 \\
\hline$\sigma=10$ & $-\quad-$ & $-\quad-$ & - & - & - & - & 3 & 1 \\
\hline$\sigma=15$ & $43 \quad 15$ & $45 \quad 56$ & 43 & 52 & 41 & 42 & 22 & 9 \\
\hline
\end{tabular}

Even though both versions of the proposed method give rise to the same detection results, the accuracy in the values of $\mu$ is higher when using the fuzzy clustering algorithm to separate mixture components. 


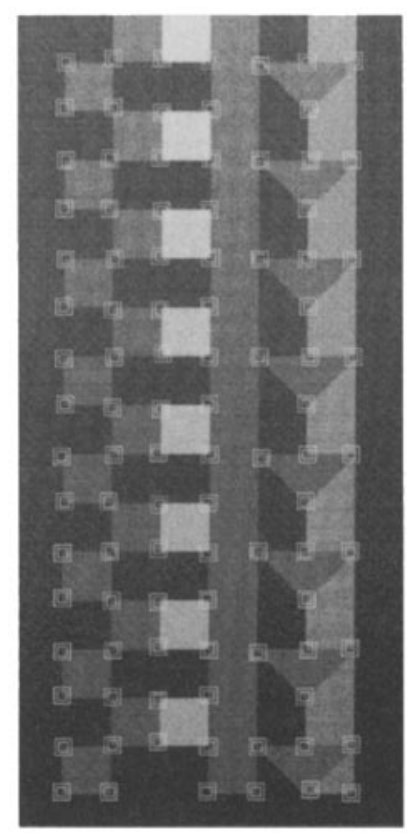

$\sigma=6$

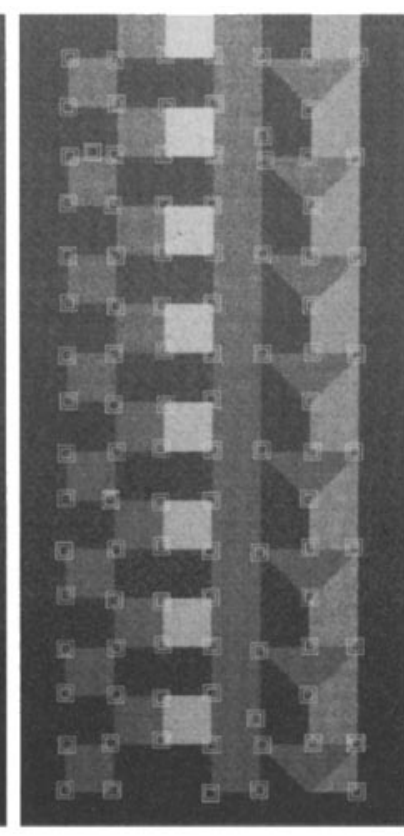

$\sigma=10$

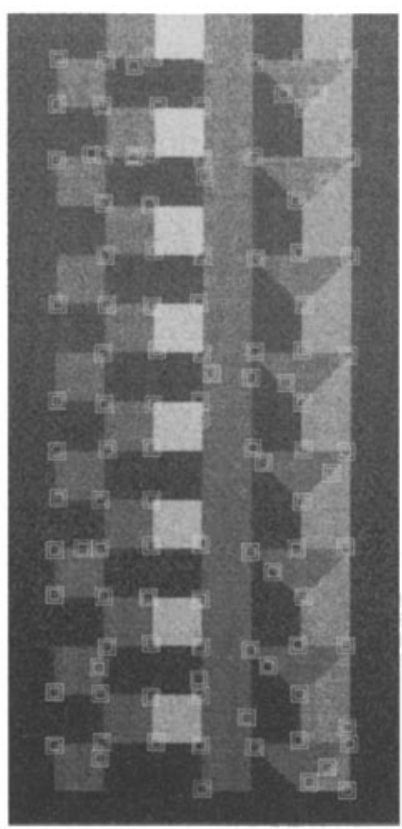

$\sigma=15$

Fig. 2. Results of detection of corners on noisy images

To quantify the accuracy of the corner detector to characterize corner points, a numerical study has been performed using the same set of synthetic images. For each detected corner, the real and estimated edge orientations are known. The accuracy of the estimators is measured by calculating the squared root of the mean squared error $(\sqrt{M S E})$. Table 2 shows that the fuzzy method always reduces the errors with respect to the crisp methodology. This improvement of the orientation estimations can also be observed graphically in Figure 3.

Table 2. Observed squared root of the mean squared error (in degrees) of the edge orientations. The fuzzy method reduce the error with respect to the crisp methodology

\begin{tabular}{|l|cc|cc|}
\hline Corner & \multicolumn{2}{|c|}{ Crisp version } & Fuzzy version \\
\cline { 2 - 5 } type & $\sigma=5 \sigma=10$ & $\sigma=5$ & $\sigma=10$ \\
\hline 2 -edges & 5.46 & 11.73 & 5.13 & 8.22 \\
3 - edges & 4.13 & 12.45 & 3.25 & 3.97 \\
4 - edges & 3.48 & 6.00 & 2.73 & 5.22 \\
\hline
\end{tabular}




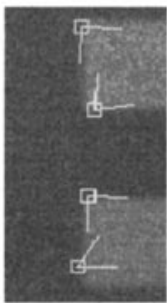

(a)

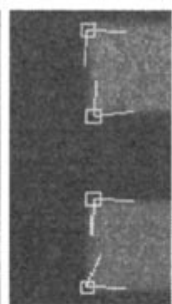

(b)

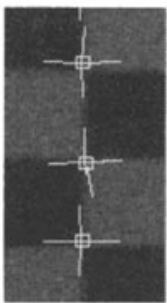

(c)

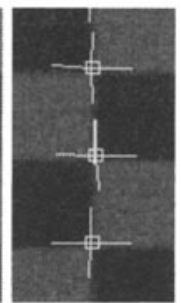

(d)

Fig. 3. Mean direction estimated by using both clustering methods: (a) and (c) crisp clustering and (b) and (d) fuzzy clustering

Finally, a more realistic experiment using images taken from ocular fundus fluorescein angiographies has been considered to detect vessel junctions (see figure 4). The goal in this case is to see, from a subjective point of view, how the proposed method performs in this (difficult) case. Angiography is a diagnosis technique used to detect ocular fundus illnesses like diabetic retinopathy; it consists on the injection of a bright liquid (contrast) into the arteries to observe later the diffusion of it along retinal vessels. Images are taken at selected instants, constituting a sequence, and accurate determination of relevant points (in particular, corners corresponding to vessel junctions) is important since it can provide the input for a registration process of the sequence based on the detection and matching of relevant points. It represents an example of a natural image in a non-controlled environment.

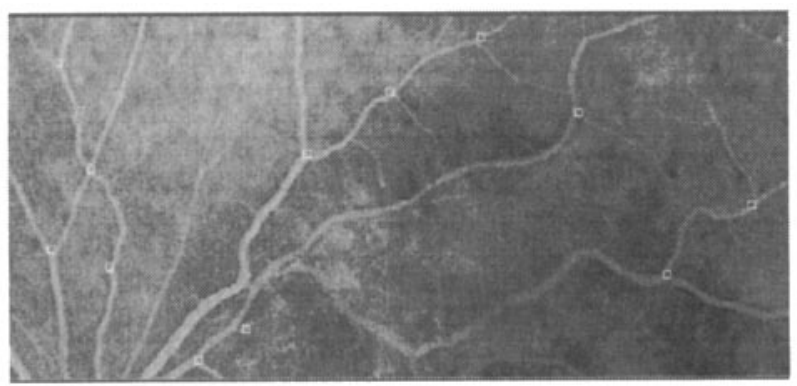

Fig. 4. Results of detection of corners on fluorescein angiographies

The influence of noise which does not manifest itself as micro-edges (like Gaussian additive noise) does not alter the uniformity of an originally uniform distribution, and it will not generally destroy the grouping tendency of a uni or multi modal von Mises distribution. This makes the algorithm particularly robust, and indeed the input parameters (gradient modulus threshold, and ac- 
cepted degree of confidence) were taken equal for all the examples, independent of the noise.

\section{Concluding Remarks}

A recently proposed method to detect 2 -D features has been modified to increase its accuracy when detecting the orientations of the edges meeting at a corner point. The method is based on the application of circular statistical techniques to the distribution of gradient or edge orientations in a circular neighbourhood of the prospective feature point.

The high confidence reached at every edge point on the hypothesis that its border orientations follow a unimodal von Mises distribution implicitly validates our initial assumption, i.e., that a junction can be modelled as a mixture of several von Mises distributions.

The use of orientations as the main source of information makes the algorithm relatively insensitive to contrast. Main novelties of the algorithm are its ability to discriminate between different types of junctions and the the capability of producing numerical estimates of orientations and their confidences.

The same method can be used to detect centers of circular symmetries by testing the uniformity of the gradient orientations. It can be easily extended to detect other $2 \mathrm{D}$ features or relevant points for specific applications.

\section{References}

1. R. Haralick and L. Shapiro. Computer and Robot Vision. Addison-Weslev, 1992.

2. P. R. Beaudet. Rotational invariant image operators. In Proc. of the Int. Conf. on Pattern Recognition, pages 579-583, 1978.

3. L. Kitchen and A. Rosenfeld. Gray level corner detection. Pattern Recognition Letters, 1:95-102, 1982.

4. J. Cooper and S. Venkatesh. Early jump-out corner detectors. IEEE Transactions on Pattern Analysis and Machine Intelligence, 15(8):823-828, 1993.

5. K. Lee and B. Zeungnam. A gray-level corner detector using fuzzy logic. Pattern Recognition Letters, 17:939-950, 1996.

6. W. Förstner et al. A fast operator for detection and precise location of distinct points, corners and centres of circular features. In Proceding of the Intercommission Conference on Fast Processing of Photogrammetruc Data., pages 281-305, 1987.

7. M.E. Díaz, J. Domingo, and G. Ayala. A gray-level 2-d feature detector using circular statistics. Pattern Recognition Letters, 18(11), 1997.

8. K.V. Mardia. Statistics of Directional Data. Academic Press, 1972.

9. G. Schou. Estimation of the concentration parameter in von mises-fisher distributions. Biometrika, 65:369-378, 1978.

10. J. Graham and G. Upton. Approximate confidence intervals for the mean direction of a von mises distribution. Biometrika, 73(2):525-7, 1986.

11. D.M. Titterington, A.F.M. Smith, and U.E. Makov. Statistical Analysis of Finite Mixture Distributions. John Wiley \& Sons, 1985.

12. J.C. Bezdek. Pattern Recognition with Fuzzy Objective Function Algorithms.

Plenum Press, 1981.

13. G.S. Watson. Goodness of fit tests on a circle. Biometrika, 49:57-63, 1962. 